\title{
Synthesis and Spectral Characterization of Some New Novel Schiff bases Derived From Hydroxy Propiophenone
}

\author{
SHAILA WAGH ${ }^{1}$ and B.R.PATIL ${ }^{2}$ \\ 1'Department of Chemistry, Adarsh College, Hingoli (M.S.) 431513 India. \\ ${ }^{2}$ Department of Chemistry, Sharda Mahavidyalaya, Parbhani. (M.S.) 431401, India. \\ *Corresponding author E-mail: sswagh16@rediffmail.com \\ http://dx.doi.org/10.13005/ojc/370335
}

(Received: April 05, 2021; Accepted: May 15, 2021)

\begin{abstract}
New Schiff bases derived by the condensation of methyl, chloro and bromo substituted 2 ' hydroxy propiophenones with aliphatic and aromatic amines have been synthesized. The Schiff bases are yellow solids with sharp melting points. The spectral characterization of these newly synthesized Schiff base ligands has been done. These Schiff bases were characterized by IR spectroscopy, Nuclear magnetic resonance spectroscopy, UV-spectroscopy and Mass spectroscopy. IR-spectra confirmed the presence of the phenolic $\mathrm{OH}$ group and azomethine group.
\end{abstract}

Keywords: Substituted hydroxy propiophenone, Aromatic amines, Aliphatic amines, NMR, Mass, Schiff base ligands.

\section{INTRODUCTION}

Schiff bases constitute the important class of organic compounds containing azomethine group $(-\mathrm{CH}=\mathrm{N}-)$ which is generally obtained by the condensation of aromatic aldehydes or ketones with amines. Nowadays research dealing with the coordination complexes of Schiff bases with various metal ions has expanded enormously. Schiff bases act as a good chelating agent ${ }^{1}$ and exhibit extraordinary properties with metal complexes. Some heterocyclic Schiff base ligands form metal chelates ${ }^{2}$ and play a very important role in medicinal chemistry. Schiff bases due to their wide range of applications becoming more and more interesting for the researchers. The Schiff bases are very much important in bioinorganic chemistry, biomedical chemistry, ${ }^{3-4}$ pharmaceutical industries, catalysis $^{5-9}$, polymer chemistry, supramolecular chemistry, catalysis, material science. They have been found to possess antimicrobial, antifungal, antimalarial, antiviral activities ${ }^{10-15}$. Recently Schiff bases having halogen groups and their metal complexes have been found to possess special interest due to their pharmacological properties. These metal complexes of Schiff base shows various biological activities ${ }^{16-17}$. These are also used as anticancer agents. Generally, ketones show less reactivity than aldehydes. A large number of Schiff bases were synthesized from hydroxy aldehydes but lesser work has been done on hydroxy propiophenone. Schiff bases act as an effective chelating agent when it has an $\mathrm{OH}$ group in the vicinity of the azomethine $(>\mathrm{C}=\mathrm{N}$-) group and forms metal complexes that possess outstanding biological properties.

This is an Open Access article licensed under a Creative Commons license: Attribution 4.0 International (CC- BY). Published by Oriental Scientific Publishing Company @ 2018 
Because of the biological importance of Schiff bases, we have synthesized some new Schiff bases from chloro hydroxy propiophenone, bromo hydroxy propiophenone, and methyl hydroxy propiophenone with benzylamine, $p$-toluidine, aniline and propylamine and these newly prepared ligands were characterized by spectral analysis.

\section{MATERIAL AND METHOD}

Chemicals used were of analytical grade without further purification. lodination of bromo and chloro 2' hydroxypropiophenone was done by using iodine and iodic acid ${ }^{18}$. Melting points were determined by an open capillary tube and were uncorrected. Thin layer chromatography was used to determine the progress of the reaction. ${ }^{1} \mathrm{H}$ NMR spectra of all the ligands were recorded in DMSO solvent using Brucker Avance Neo $500 \mathrm{MHz}$ spectrophotometer, FTIR spectra were recorded on Shimadzu spectrometer and the mass spectra was recorded on Q-TOFMicromass (ESI-MS). UV-spectra were recorded on a W-1800 series spectrometer.

\section{General Procedure for the synthesis of Schiff bases}

All the Schiff base ligands were synthesized by refluxing substituted hydroxy propiophenone
$(0.01 \mathrm{M})$ with different aliphatic and aromatic amines $(0.01 \mathrm{M})$ for 3-5 hours. The reaction was monitored after the regular interval by TLC. When the reaction was complete, cooled the resulting mixture at room temperature and then transferred in ice-cold water. The yellow product separated which was then recrystallized by hot ethanol.

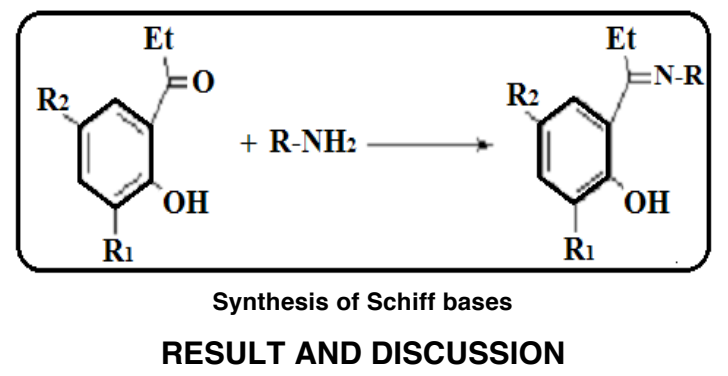

The analytical and physical data of Schiff base ligand are given in the following Table 1. All the ligands were yellow colored, solid and stable at room temperature. The UV-spectra of the ligands showed the strong absorption bands at 330-350 $\mathrm{nm}$ and $280-290 \mathrm{~nm}$ which were attributed to $n-n^{*}$ and $\pi-\pi^{\star}$ transitions respectively. Strong IR bands were observed in the range $3000-3100 \mathrm{~cm}^{-1}$ which is the characteristic of the phenolic $\mathrm{OH}$ group. The details of the spectroscopic data are illustrated below the table.

Table 1: Physical and Analytical Data of Schiff base Ligand

\begin{tabular}{|c|c|c|c|c|c|c|c|c|}
\hline \multirow[t]{2}{*}{ Ligand } & \multirow[t]{2}{*}{$\mathrm{R}_{1}$} & \multirow[t]{2}{*}{$\mathrm{R}_{2}$} & \multirow[t]{2}{*}{$\mathrm{R}$} & \multirow{2}{*}{$\begin{array}{l}\text { Melting Point } \\
\qquad\left({ }^{\circ} \mathrm{C}\right)\end{array}$} & \multirow{2}{*}{$\begin{array}{l}\text { Yield } \\
(\%)\end{array}$} & \multicolumn{3}{|c|}{ Elemental Analysis (\%) calculated (found) } \\
\hline & & & & & & $\mathrm{C}$ & $\mathrm{H}$ & $\mathrm{N}$ \\
\hline $2 a$ & $\mathrm{H}$ & $\mathrm{Br}$ & $\mathrm{C}_{6} \mathrm{H}_{5} \mathrm{CH}_{2}$ & 110 & 68 & $60.39(60.56)$ & $5.10(5.04)$ & $4.35(4.41)$ \\
\hline $2 b$ & 1 & $\mathrm{Br}$ & $\mathrm{C}_{6} \mathrm{H}_{5}-\mathrm{CH}_{2}$ & 122 & 62 & $36.39(36.54)$ & $3.72(3.80)$ & $3.44(3.55)$ \\
\hline $3 a$ & $\mathrm{H}$ & $\mathrm{CH}_{3}$ & $\mathrm{C}_{6} \mathrm{H}_{5}$ & 131 & 66 & $80.20(80.33)$ & $7.16(7.11)$ & $5.75(5.85)$ \\
\hline $3 b$ & 1 & $\mathrm{Br}$ & $\mathrm{C}_{6} \mathrm{H}_{5}$ & 130 & 55 & $41.89(41.95)$ & $3.08(3.03)$ & $3.36(3.26)$ \\
\hline $4 a$ & $\mathrm{H}$ & $\mathrm{CH}_{3}$ & $\mathrm{C}_{6} \mathrm{H}_{4}-\mathrm{OCH}_{3}$ & 85 & 54 & $80.50(80.63)$ & $7.56(7.50)$ & $5.43(5.53)$ \\
\hline $4 b$ & I & $\mathrm{Cl}$ & $\mathrm{C}_{6} \mathrm{H}_{4}-\mathrm{OCH}_{3}$ & 125 & 55 & $48.14(48.06)$ & $3.82(3.75)$ & $3.55(3.5)$ \\
\hline $5 a$ & $\mathrm{H}$ & $\mathrm{Br}$ & $\mathrm{n}-\mathrm{C}_{3} \mathrm{H}_{7}$ & 82 & 54 & $53.35(53.53)$ & $5.77(5.94)$ & $5.08(5.20)$ \\
\hline $5 b$ & $\mathrm{H}$ & $\mathrm{Cl}$ & $\mathrm{n}-\mathrm{C}_{3} \mathrm{H}_{7}$ & 72 & 61 & $70.32(70.20)$ & $5.91(5.85)$ & $5.22(5.11)$ \\
\hline $6 a$ & $\mathrm{H}$ & $\mathrm{Cl}$ & Iso- $\mathrm{C}_{3} \mathrm{H}_{7}$ & 90 & 65 & $70.32(70.20)$ & $5.91(5.85)$ & $5.22(5.11)$ \\
\hline $6 b$ & 1 & $\mathrm{Cl}$ & Iso- $\mathrm{C}_{3} \mathrm{H}_{7}$ & 110 & 40 & $40.89(41.08)$ & $4.23(4.27)$ & 3.67(3.99) \\
\hline
\end{tabular}

2a) 2-(1-(benzyl imino) propyl)4-bromophenol

IR (KBr): $3028.37(\mathrm{OH}), 1604.85$ $(\mathrm{C}=\mathrm{N}), 1284.65(\mathrm{C}-\mathrm{O}) \mathrm{cm}^{-1} \cdot{ }^{1} \mathrm{H}$ NMR(DMSO):- $\delta$ 1.16(t,3H), 2.97 (q, 2H), 4.88(s, 2H), 6.63-7.79(m, Ar-H), 16.86(s, 1H, OH)ppm Mass:- m/z-317M+.

2b)2-(1-(benzyl imino) propyl)4-bromo-6-iodo phenol

$\mathrm{IR}(\mathrm{KBr}): 3078.52(\mathrm{OH}), 1612.56(\mathrm{C}=\mathrm{N}), 1284$
$.65(\mathrm{C}-\mathrm{O}) \mathrm{cm}^{-1} .{ }^{1} \mathrm{H}$ NMR(DMSO):- $\delta 1.07(\mathrm{t}, 3 \mathrm{H}), 3.18 \mathrm{v}$ (q, 2H), 3.32(s, 2H), 6.63-7.79(m, Ar-H), $12.97(\mathrm{~s}, 1 \mathrm{H}$, $\mathrm{OH})$ ppm Mass:-m/z-443 $\mathrm{M}^{+}$.

\section{3a)4-methyl-2-(1-phenylimino)propyl phenol}

IR(KBr): 3055.38(OH), 1597.13(C=N), 1203.63(C-O) $\mathrm{cm}^{-1} .{ }^{1} \mathrm{HNMR}(\mathrm{DMSO}):-\delta 1.16(\mathrm{t}, 3 \mathrm{H})$, 2.23(q, 2H), 6.63-7.79(m, Ar-H),14.45(s, 1H, OH) ppm Mass:-m/z-239M+ 
3b)4-bromo-2-iodo-6-(1-phenylimino)propyl phenol

$\mathrm{IR}(\mathrm{KBr}): 3045.38(\mathrm{OH}), 1651.14(\mathrm{C}=\mathrm{N}), 12$ 73.07(C-O) $\mathrm{cm}^{-1} .{ }^{1} \mathrm{H}$ NMR(DMSO) :- $\delta 1.17(\mathrm{t}, 3 \mathrm{H})$, 2.50(q, 2H), 7.35-7.84(m, Ar-H), 17.78(s,1H,OH)ppm Mass:-m/z-429 $\mathrm{M}^{+}$

4a) 2-(1-p -tosylimino) propyl)4-methyl phenol $\mathrm{IR}(\mathrm{KBr}): 3065.51(\mathrm{OH}), 1643.42(\mathrm{C}=\mathrm{N})$, 1273.07(C-O) $\mathrm{cm}^{-1}{ }^{1}{ }^{1} \mathrm{H}$ NMR(DMSO) :- $\delta 1.21(\mathrm{t}, 3 \mathrm{H})$, 2.99(q, 2H), 2.35(s, 6H, Ar- $\mathrm{CH}_{3}$ ), 7.08-7.30(m, Ar$\mathrm{H}), 16.00(\mathrm{~s}, 1 \mathrm{H}, \mathrm{OH}) \mathrm{ppm}$ Mass:-m/z-253M+

\section{4b)2-(1-p-tosylimino) propyl)4-chloro-6-iodo phenol}

$\mathrm{IR}(\mathrm{KBr})$ : 3063.09(OH), 1651.14(C=N),1265. $36(\mathrm{C}-\mathrm{O}) \mathrm{cm}^{-1} .{ }^{1} \mathrm{H}$ NMR(DMSO) :- $\delta 1.16(\mathrm{t}, 3 \mathrm{H})$, 2.02(q, 2H), 1.01(s,3H), 6.67-7.79(m, Ar-H), 15.55(s, $1 \mathrm{H}, \mathrm{OH})$ ppm Mass:-m/z-399 M+

5a)4-bromo-2-(E)-1-(propyl imino) propyl phenol $\mathrm{IR}(\mathrm{KBr}): 3070.81(\mathrm{OH}), 1643.42(\mathrm{C}=\mathrm{N}), 1273$. $07(\mathrm{C}-\mathrm{O}) \mathrm{cm}^{-1}{ }^{1}{ }^{1} \mathrm{H}$ NMR(DMSO) :- $\delta 1.01(\mathrm{t}, 3 \mathrm{H}), 1.16$ $(\mathrm{t}, 3 \mathrm{H}), 2.01(\mathrm{~m}, 2 \mathrm{H}), 3.89(\mathrm{t}, 2 \mathrm{H})$, 6.45-8.19 (m, Ar-H), $15.86(\mathrm{~s}, 1 \mathrm{H}, \mathrm{OH}) \mathrm{ppm}$ Mass:-m/z-269 M+

5b) 4-chloro-2- (E)-1-(propyl imino) propyl phenol $\mathrm{IR}(\mathrm{KBr}): 3068.52(\mathrm{OH}), 1612.56(\mathrm{C}=\mathrm{N})$, 1262.43(C-O) $\mathrm{cm}^{-1}{ }^{1}{ }^{1} \mathrm{H}$ NMR(DMSO) :- $\delta 1.01(\mathrm{t}, 3 \mathrm{H})$, 1.11 (sex, 2H), 2.83(t, 2H), 8.13-8.19(m, Ar-H), 17.10(s, 1H, OH)ppm Mass:-m/z-225 M+

6a) 4-chloro-2-(1-isopropyl imino) propyl phenol $\mathrm{IR}(\mathrm{KBr}): 3075.55(\mathrm{OH}), 1602.16(\mathrm{C}=\mathrm{N}), 1284$ 65 (C-O) $\mathrm{cm}^{-1}{ }^{1}{ }^{1} \mathrm{H}$ NMR(DMSO) :- $\delta 1.03(\mathrm{t}, 3 \mathrm{H}), 1.95$ (q, $2 \mathrm{H}), 3.48(\mathrm{~m}, 1 \mathrm{H}), 6.70-7.48(\mathrm{~m}, \mathrm{Ar}-\mathrm{H}), 15.45$ (s, $1 \mathrm{H}, \mathrm{OH}$ )ppm Mass:-m/z-225 M+

6b) 4-chloro-2-iodo 6-(1-(isopropyl imino) propyl phenol

$\mathrm{IR}(\mathrm{KBr}): 3078.52(\mathrm{OH}), 1612.56(\mathrm{C}=\mathrm{N}), 12$ 84.65(C-O) $\mathrm{cm}^{-1}{ }^{1}{ }^{1} \mathrm{H}$ NMR(DMSO) :- $\delta 1.16(\mathrm{t}, 3 \mathrm{H})$, 2.04(q, 2H), 3.87 (m, 1H), 6.87-7.67(m, Ar-H), 16.06 (s, $1 \mathrm{H}, \mathrm{OH})$ ppm Mass:-m/z-350 $\mathrm{M}^{+}$

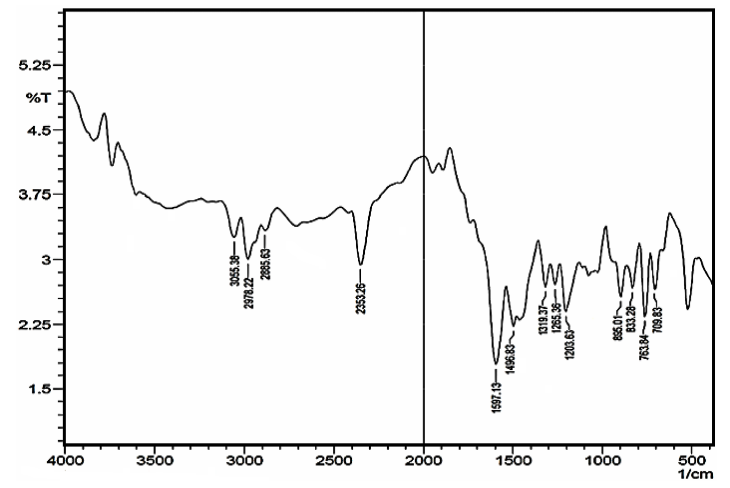

Fig. 1. IR spectra of $3 a$

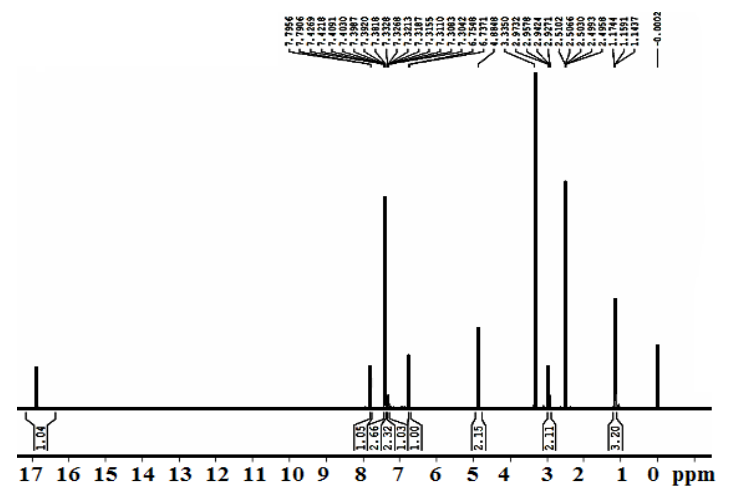

Fig. 2. ${ }^{1} \mathrm{H}$ NMR spectra of $2 \mathrm{a}$

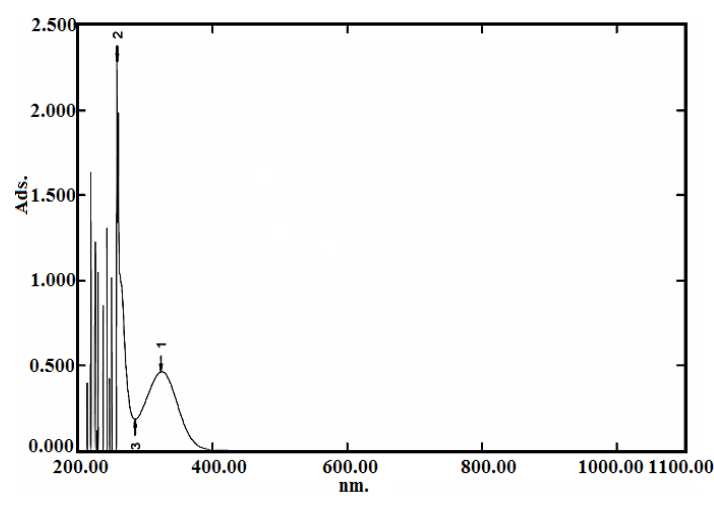

Fig. 3. UV spectra of $2 b$

\section{CONCLUSION}

New Schiff base ligands were synthesized by reacting substituted 2 ' hydroxy propiophenone with different aromatic and aliphatic amines. All the ligands showed a strong IR band at 3000$3200 \mathrm{~cm}^{-1}$ for phenolic $-\mathrm{OH}$, a band at 1684-1595 $\mathrm{cm}^{-1}$ for $\mathrm{C}=\mathrm{N}$ stretch. All the structures were confirmed by NMR and mass spectra. NMR studies showed the presence of $\mathrm{OH}$ peak at 12 to $17 \mathrm{ppm}$ in all the ligands. 


\section{ACKNOWLEDGEMENT}

The authors are thankful to Principal, D. S. M. College, Parbhani and Principal, Adarsh College, Hingoli, and the Director, SAIF Punjab
University, Chandigarh for providing spectral data.

\section{Conflicts of Interest}

The authors declare no conflict of interest.

\section{REFERENCES}

1. Krishnapriya K.R.; Kandaswamy M., Polyhedron., 2005, 24,113.

2. Abd El-Halim H. F. Mohamed G. G.; Anwar M. N., Appl Organometal Chem., 2017, e3899.

3. Saito H.; Hoffmann A.S. Ogazoa H.I.J.; Bioact. Compd. Polym., 2007, 22, 589.

4. In G.; Kim Y and Choi J., Bull. Korean Chem. Soc., 2008, 29(5), 969.

5. Panja A.; Shaikh N.; Ali M.; Vojtisek P.; Banerjee P., Polyhedron., 2003, 22, 1191.

6. Djebbar S. S.; Benali B.O.; Deloume J. P.; Trans. Metal Chem., 1998, 23, 443.

7. Xishi Tai Xianhong Y.; Qiang C.; Minya T.; Molecules., 2003, 8, 49.

8. Wang K.; Wedeking K.; Zuo W.; Zhang D.; Wen-Hua Sun, J.Organomet Chem., 2008, 693(6), 1073.

9. $\quad$ Long Z.; Wu B.; Yang P.; Gang Li, Liu Y.; XiaoJuan Y., J. Organomet. Chem., 2009, 694(32), 3793.

10. Ommenya, F. K.; Nyawade, E. A.; Andala, D. $M$ and Kinyua, J., Journal of Chemistry, 2020,
1745236.

11. Spinu C.; Pleniceanu M.; Tigae C. Turkish J Chem., 2008, 32(4), 487-493.

12. Zafar H.; KareemA.; Sherwani A., J Photochem Photobiol B Biol., 2015, 142, 8-19.

13. Hueso-Urena F.; Illan-Cabeza NA.; MorenoCarretero MN.; Martinez-Martos JM.; Ramnirez-Exposito MJ. J. Inorg. Biochem., 2003, 94(4), 326-334.

14. Abd El-halim H. F.; Omar M. M.; Mohamed G. G., Spectrochim. Acta, Part A, 2011, 78, 36-44.

15. Hameed A.; Al-Rashida M.; Uroos M.; Abid Ali S.; Khan K. M.; Expert Opin. Ther. Pat., 2017, 27, 63-79.

16. Samina K.T.; Abhijit A.Y.; Ratnamala S.B. J. Mol. Struct., 2018, 1152, 223-231.

17. Kursunlu A.N.; Guler E.; Sevgi F.; Ozkalp B., J. Mol. Struct., 2013, 1048, 476-481.

18. Patil B.R.; Bhusare S.R., Pawar Tetrahedron Letters, 2005, 46(42), 7179-7181. 